\title{
Evaluación de la composición nutricional de los menús expendidos en restaurantes: Necesidad de nueva información
}

\author{
Evaluation of the nutritional composition of menus sold \\ in restaurants: Need for new information.
}

Sr. Editor

En la actualidad se ha mostrado una alta variabilidad enla ingesta de alimentos adquiridos fuera del hogar por parte de la población a nivel mundial. Ello, debido a un cambio en los hábitos alimentarios a lo largo del tiempo ${ }^{1,2}$. Estos cambios se deben a que la población adopta costumbres que pueden ser influenciadas por la publicidad. Dichas costumbres vienen de factores modificables como la urbanización, el ingreso económico y el incremento en la disponibilidad y acceso a los alimentos ${ }^{3,4}$. Cabe destacar además, un aumento en el consumo de alimentos procesados, debido al incremento en las horas laborales por parte de las familias, que a su vez explica el consumo de alimentos fuera del hogar'.

Otros factores que influyen en la adquisición de alimentos fuera del hogar, son el incremento en la oferta gastronómica y la falta de tiempo con que cuenta la población joven y adulta, debido al incremento en el número de horas de trabajo. Todo esto induce a conductas alimentarias no adecuadas y/o modificaciones en la ingesta alimentaria tanto en cantidad como calidad, según el lugar donde sea adquirido $^{5,6}$.

Por tal motivo, consideramos importante que exista y/o se pueda brindar mayor información a la población, acerca de la cantidad y calidad de la comida consumida fuera del hogar. La calidad de la dieta se refiere a la concentración de macronutrientes y micronutrientes que contiene una ración y la biodisponibilidad de estos nutrientes ${ }^{5}$, la cual puede ser medida mediante métodos directos e indirectos, tales como el análisis químico proximal y la evaluación del contenido de nutrientes en las recetas, respectivamente ${ }^{7}$.

Finalmente, al existir limitada información de la calidad y valor nutricional de los alimentos que puede contener un plato de comida, consideramos que la comunidad científica e industria de alimentos, deben hacer más estudios sobre el tema, donde intervengan las entidades públicas, tanto a nivel local como internacional, para así contribuir a la salud pública de cada país. En el Perú, la "Encuesta para medir la Composición Nutricional de los Principales Alimentos Consumidos Fuera del Hogar" (ENCONUT) ${ }^{2}$ ha brindado
Mariale Benavides ${ }^{1}$, Daniela Vizarreta $D^{\prime}$, Jorge L. Maguiña' .

1. Facultad de Ciencias de la Salud, Universidad Peruana de Ciencias Aplicada.

Dirigir correspondencia a: Daniela Vizarreta Donayre. Universidad Peruana de Ciencias Aplicadas, Lima, Perú. Escuela de Nutrición y Dietética. Teléfono: 957328673. E-mail: dani.viza.25@gmail.com

Este trabajo fue recibido el 05 de mayo de 2017. Aceptado con modificaciones el 12 de julio de 2017 y aceptado para serpublicado el 14 de julio de 2017.

información sobre estos aspectos. Sin embargo, un mayor detalle en sus análisis y futura publicaciónes aún es un tema pendiente.

\section{BIBLIOGRAFÍA}

1. Koplan J, Liverman CT, Kraak VI. Preventing Childhood Obesity: Health in the Balance. National Academies Press; 434 p. 2005.

2. INEI. Survey to measure the nutritional composition of principal foods consumed outside the home [Internet]. [cited 2017 May 5]. Available from: http://iinei.inei.gob.pe/iinei/ srienaho/Descarga/DocumentosMetodologicos/2013-48/ INFORME_FINAL_ENCONUT2013.pdf 
3. FAO. Chapter 5: Population, food, nutrition and family planning [Internet]. [cited 2017 May 5]. Available from: http://www. fao.org/docrep/006/w0073s/w0073s09.htm

4. Villa P. AL. Food preferences in the city of Medellín, Colombia | Montoya | Health and Society [Internet]. [cited 2017 May 5]. Available from: http://www.revistas.usp.br/sausoc/article/ view/123062/119445

5. MINSA. GUIDE: Calculation of the nutritional composition of preparations distributed in the wawa wasi national program [Internet]. [cited 2017 May 5]. Available from: http://bvs. minsa.gob.pe/local/minsa/1371.pdf

6. Kamisha Hamilton Escoto, Melissa Nelson Laska, Nicole Larson, Dianne Neumark-Sztainer, Peter J. Hannan. Work Hours and Perceived Time Barriers to Healthful Eating Among Young Adults [Internet]. [cited 2017 May 5]. Available from: https://www.ncbi.n/m.nih.gov/pmc/articles/PMC3464955/ pdf/nihms373265.pdf

7. D.A.T Southgate HG. Food composition data: Obtaining, managing and utilizing [Internet]. [cited 2017 May 5]. Available from: http://www.fao.org/3/a-y4705s.pdf 